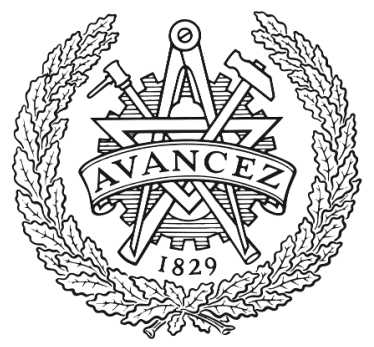

CHALMERS

UNIVERSITY OF TECHNOLOGY

\title{
Improvement of DPSK transmission by using convolutional error correction coding
}

Downloaded from: https://research.chalmers.se, 2023-04-26 07:47 UTC

Citation for the original published paper (version of record):

Wuth, T., Agrell, E., Karlsson, M. (2005). Improvement of DPSK transmission by using convolutional error correction coding. Optical Fiber Communication Conference, 2005. Technical Digest. OFC/NFOEC, 4: 534-536. http://dx.doi.org/10.1109/OFC.2005.193044

N.B. When citing this work, cite the original published paper. 


\title{
Improvement of DPSK Transmission by Using Convolutional Error Correction Coding
}

\author{
Torsten Wuth, Erik Agrell \\ Dept. of Signals and Systems, Chalmers Univ. of Technology, SE-412 96 Gothenburg, Sweden \\ email: torsten.wuth@ieee.org, agrell@s2.chalmers.se \\ Magnus Karlsson \\ Photonics Lab., MC2, Chalmers Univ. of Technology, SE-412 96 Gothenburg, Sweden \\ email: magnus.karlsson@mc2.chalmers.se
}

\begin{abstract}
In this paper we quantify the improvement in the transmission quality for DPSK transmission by using convolutional error correction coding. To avoid bandwidth-limitation problems from e.g. chromatic dispersion the convolutional coding is combined with bandwidth efficient modulation.

(c) 2005 Optical Society of America

OCIS codes: (060.2330) Fiber Optics Communications; 060.4080 Modulation
\end{abstract}

\section{Introduction}

The rapid development of fiber optic transmission, with increasing channel rates and transmission distances is forcing the development of not only transmission technology, but also novel efficient modulation formats and channel coding schemes. Therefore we will investigate the opportunity to decrease the bit error rate (BER) of a return-to-zero differential phase shift keying (RZ-DPSK) transmission over standard single mode fiber (SMF) by applying convolutional line coding [1]. The advantage of convolutional coding is (i) a low memory effort, which translates into a low gate count and low power consumption [2]), (ii) that it works on a continuous data stream and hard as well as soft decision decoding is possible. The drawback is the high code rate. We investigate in this paper a code rate of $1 / 2$, which means an overhead of $100 \%$. To avoid a strong increase of distortion caused by group velocity dispersion (GVD), we intend to compare coded and uncoded systems with the same information bit rate and bandwidth. The modulation schemes are return-to-zero differential quadrature phase shift keying (RZ-DQPSK) [3] and RZASK/DSPK modulation [4], which is a combination of amplitude shift keying (ASK) and different phase shift keying (DPSK). For uncoded transmission, RZ-DPSK modulation is used as a benchmark[5]. We believe this is the first time convolutional coding together with hard and soft decision decoding is evaluated for optical communications.

The used convolutional code is a $(1167,1545)$-code with a constraint length of 10 [6, p. 222] (the constraint length indicates the maximum number of bits in a single output stream that can be influenced by a single input bit).

\section{Simulation setup}

The optical transmission line is modelled as follows: After being generated in the transmitter, the signal is propagated linearly through varying lengths of standard singlemode fiber with a dispersion of $17 \mathrm{ps} / \mathrm{nm} \mathrm{km}$. The carrier wavelength of the signal is $1550 \mathrm{~nm}$. Next the signal is fed into a variable optical attenuator (VOA), followed by an optical preamplifier (an erbium-doped fiber amplifier, EDFA). By varying the attenuation of the VOA, the input power into the EDFA is changed, and this causes also a change in the optical signal-to-noise ratio. It is assumed that the signal and the noise has the same polarization. The EDFA gain is $G=30 \mathrm{~dB}$ and the noise figure is $F=5 \mathrm{~dB}$. After amplification, the signal passes an optical, Gaussian-shaped bandpass filter (BPF) and is detected in the receiver. The information payload is in all cases $10 \mathrm{~Gb} / \mathrm{s}$.

The RZ-DQPSK transmitter setup and the signal in the complex plane is displayed in Fig. 1 (right). The RZDQPSK transmitter consists of three stages. In the first two stages a RZ-DPSK signal is formed by two Mach-Zehnder modulators (MZM). In the third stage four symbols are generated, out of the binary PSK signal, by a subsequent phase modulator (PM). The two precoded data streams which are applied to the first MZM and the PM, are the output signal of a DQPSK precoder. The precoder is in turn fed by the output sequences of the convolutional encoder. The signal constellation is displayed in the inset of the transmitter setup. The RZ-DPSK transmitter consists of the first two stages of the RZ-DQPSK transmitter. In the complex plane the signal occupies the two signal points on the real axis. The RZ-ASK/DPSK transmitter consists also of three stages. In every stage a Mach-Zehnder modulator, 
RZ-DQPSK

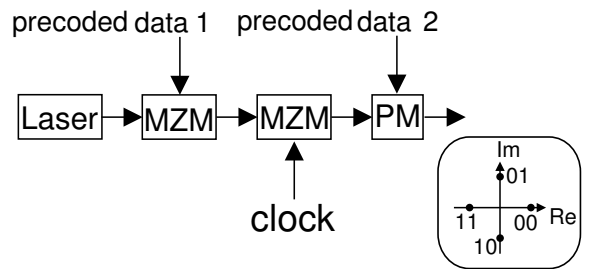

RZ-ASK/DPSK

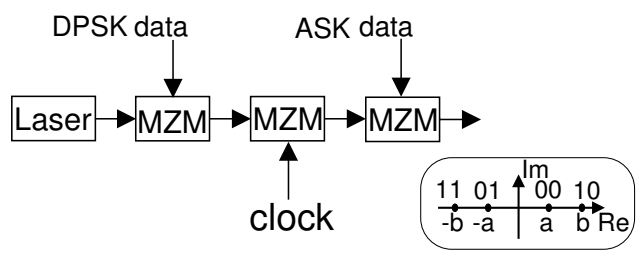

Fig. 1. RZ-DQPSK transmitter (left) and RZ-ASK/DPSK transmitter (right). Insets: Signal constellation of the used modulation formats.

in push-pull configuration, is used. In the first stage, the continuous wave signal from the laser is phase modulated with the DPSK data. The information is modulated to the phase 0 or $\pi$. The second stage is responsible for RZ pulse shaping with a duty cycle of 0.5 . Finally the ASK data is modulated in the third stage. The amplitude levels are $a$ (for a ' 0 ') and $b$ (for a '1'). With this configuration the RZ-ASK/DPSK signal is generated. The signal constellation in the complex plane is also shown in Fig. 1. To obtain similar bit error rate (BER) in the ASK and DPSK branch of the RZ-ASK/DPSK signal, the ratio between $a$ and $b$ is numerically found to be $a / b=0.398$. In the case of coded transmission, the ASK and DPSK data are the output data of the convolutional encoder.
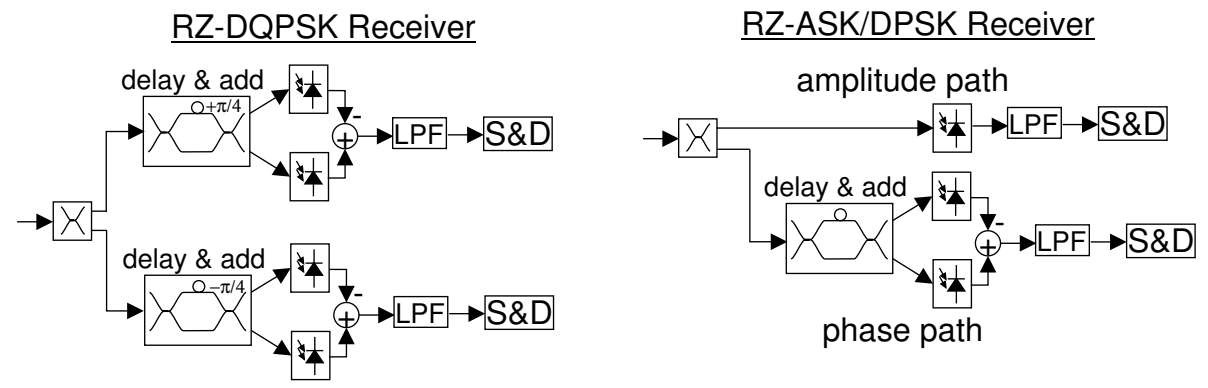

Fig. 2. RZ-DQPSK receiver (left) and RZ-ASK/DPSK receiver (right).

The used receivers for the RZ-DQPSK and the RZ-ASK/DPSK signals are shown in Fig. 2. In the DQPSK-receiver (Fig 2, left) we feed the signal in to two delay \& add circuits, each consisting of two concatenated $3 \mathrm{~dB}$ couplers, connected with a differential delay of one symbol $\pm \pi / 4$ [7]. Both outputs of the $3 \mathrm{~dB}$ coupler are used to feed a balanced receiver, filtered by an electrical lowpass filter (LPF), sampled and decoded. The LPF is a third order butterworth filter with a $3 \mathrm{~dB}$ cutoff frequency of $7 \mathrm{GHz}$. In the RZ-ASK/DPSK receiver (Fig.2 right) the ASK signal is received by a photodiode, and after passing an electrical lowpass filter (LPF), the signal is supplied to a sampling and decision (S\&D) module. The phase path is similar to one of the paths of the RZ-DQPSK receiver, without the phase shift of $\pi / 4$. The combined output of the photodiodes is filtered by an electrical low pass filter. The filtered signal is then sampled and detected. The receiver of the RZ-DPSK signal consist only the phase path of the RZ-ASK/DPSK receiver.

In the case of coded transmission, the detected data is fed into a Viterbi hard and soft decision decoder. The soft decision decoder operates on analogue values, and according to [1] the use of an analog-to-digital converter with three or four bits resolution would result in a low additional penalty of 0.5 and $0.2 \mathrm{~dB}$, respectively.

The simulations used random data and was continued for different receiver power levels until 30-100 errors was obtained. The BER levels below $10^{-8}$ required several weeks of computation time on a 17 -node computer cluster.

\section{Results and discussion}

The simulation results of the transmission with and without fiber dispersion are displayed in Fig. 3. The improvement of convolutional coding with soft and hard decision without dispersive fiber transmission is displayed in the left figure, and the influence of fiber dispersion shown in the mid and right of Fig 3. To get the coding gain we compare the receiver input power at a BER of $10^{-8}$ with the RZ-DPSK as a reference at $-43.6 \mathrm{dBm}$. The corresponding power for non-return to zero ASK is $-39.8 \mathrm{dBm}$ (not shown in the figure). By using the RZ-ASK/DPSK transmission scheme we find a coding gain of $0.7 \mathrm{~dB}$ for hard decision decoding, and about $3.4 \mathrm{~dB}$ for soft decision decoding. 
For the RZ-DQPSK transmission scheme the coding gain is slightly better, being about $2.2 \mathrm{~dB}$ for hard decision and about $4.4 \mathrm{~dB}$ for soft decision, as compared to the DPSK. We can thus see that the RZ-DQPSK performs better than the RZ-ASK/DPSK, which we attribute to the larger separation between the modulation levels in signal space for DQPSK.

When fiber dispersion is included in the simulation setup, we compare the necessary receiver power to get a BER of $10^{-5}$. For the RZ-DQPSK transmission scheme the back-to-back coding gain is about $2 \mathrm{~dB}$ for hard decision and $3.9 \mathrm{~dB}$ for soft decision. This improvement of the coding gain increases after $62.5 \mathrm{~km}$ to $2.6 \mathrm{~dB}$ for hard and to 5.2 $\mathrm{dB}$ for soft decision. It is also possible, by using soft decision, to increase the transmission length to $75 \mathrm{~km}$, with a penalty of about $4 \mathrm{~dB}$ in comparison to uncoded DPSK transmission back-to-back. Uncoded transmission of $75 \mathrm{~km}$ fiber length is not possible due to bit-error-rate floors.

For RZ-ASK/DPSK and hard decision the improvement of transmission quality is negligible. The coding gain is about $0.1 \mathrm{~dB}$ and $0.4 \mathrm{~dB}$ depending on the fiber length. With soft decision decoding the coding gain is about $2.5 \mathrm{~dB}$ at a fiber length of $0 \mathrm{~km}$. After $62.5 \mathrm{~km}$ the coding gain have increased to $3.7 \mathrm{~dB}$. Also here, a transmission length of $75 \mathrm{~km}$ is possible only by using convolutional coding and soft decision decoding.
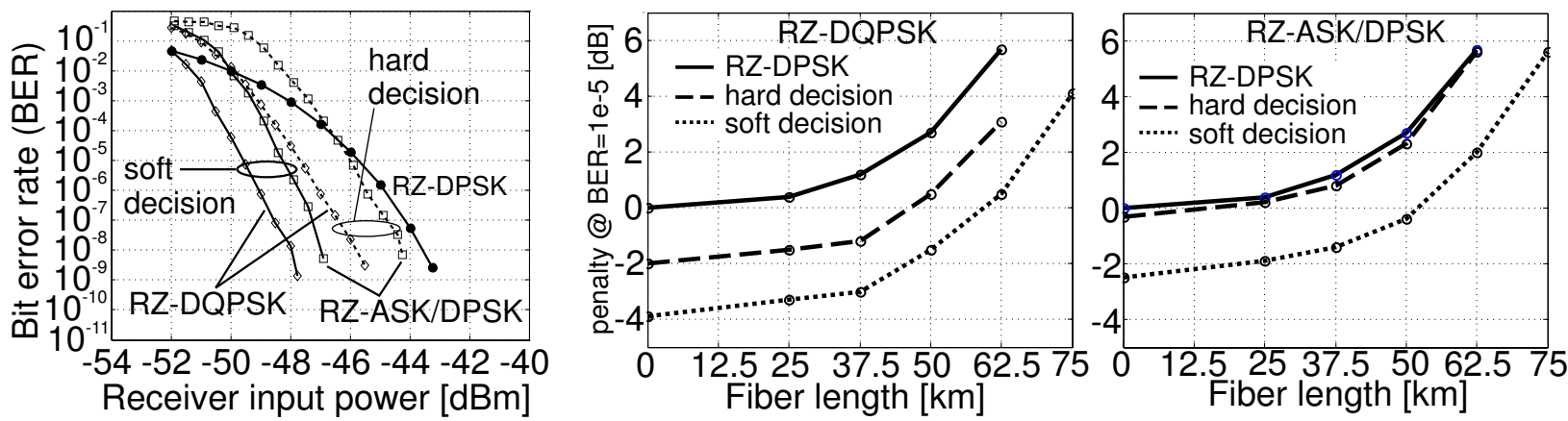

Fig. 3. BER curves back-to-back for coded and uncoded transmission with hard/soft decision (left). BER vs dispersive fiber length for RZ-DQPSK receiver (mid) and RZ-ASK/DPSK receiver (right).

\section{Conclusion}

The transmission improvement by using convolutional coding in the presence of ASE and chromatic dispersion have been determined numerically at $10 \mathrm{~Gb} / \mathrm{s}$. Therefore two different bandwidth efficient modulation schemes (RZDQPSK and RZ-ASK/DPSK) are used for transmitting the coded data. Hard and soft decision decoding is used. We have used RZ-DPSK transmission as the reference modulation scheme. The RZ-DQPSK transmission scheme offers a clearly better coding gain than the RZ-ASK/DPSK scheme, being about $2.2 \mathrm{~dB}$ for hard decision and and $4.4 \mathrm{~dB}$ for soft decision at a BER of $10^{-8}$. The RZ-ASK/DPSK format offers a coding gain of about $0.7 \mathrm{~dB}$ for hard decision and $3.4 \mathrm{~dB}$ for soft decision decoding. The coding gain increases when fiber dispersion is included in the transmission model, and SMF transmission lengths of $75 \mathrm{~km}$ can only be reached by using soft decision decoding for both bandwidth efficient modulation schemes.

\section{References}

1. H. Bülow et al, Optical Trellis-Coded Modulation, Proc. of Optical Fiber Communication Conference, OFC 2004, paper WM5, 2004.

2. K. Seki at al, Single-chip FEC codec LSI using iterative CSOC decoder for $10 \mathrm{~Gb} / \mathrm{s}$ long-haul optical transmisison systems, IEEE Custom Integrated Circuits Conference, May 12-15, 2002, Orlando, USA, pp. 155-158

3. C. Wree et al, RZ-DQPSK Format with High Spectral Efficiency and High Robustness Towards Fiber Nonlinearities, Proc. of European Conference on Optical Communication, ECOC 2002, paper 9.6.6, 2002.

4. M. Ohm, J. Speidel, Quaternary Optical ASK-DPSK and Receivers with Direct Detection, IEEE Photonics Technology Letters, Vol. 15, No. 1, pp. 159-161, 2003.

5. J. Leibrich et al, CF-RZ-DPSK for Suppression of XPM on Dispersion-Managed Long-Haul Optical WDM Transmission on Standard Single-Mode Fiber, Photonics Technology Letters, Vol. 14, pp 155-157, 2002

6. R. B. Wells, Applied Coding and Information Theory for Engineers, Pentrice Hall, Inc., 1999.

7. R.A. Griffin et al, Optical Differential Quadrature Phase-Shift Key (oDPSK) for High Capacity Optical Transmission, Proc. of Optical Fiber Communication Conference, OFC 2002, paper WX6, 2002. 